\title{
Detecting hydrologic deformation using GRACE and GPS
}

\author{
P. Tregoning, ${ }^{1}$ C. Watson, ${ }^{2}$ G. Ramillien, ${ }^{3}$ H. McQueen, ${ }^{1}$ and J. Zhang ${ }^{1}$ \\ Received 17 April 2009; revised 28 May 2009; accepted 2 June 2009; published 7 August 2009.
}

[1] Hydrological processes cause variations in gravitational potential and surface deformations, both of which are detectable using space geodetic techniques. We computed elastic deformation using continental water load estimates derived from the Gravity Recovery and Climate Experiment and compared to 3D deformation estimated from GPS observations. The agreement is very good in areas where large hydrologic signals occur over broad spatial scales, with correlation in horizontal components as high as 0.9 . Agreement is also observed at smaller scales, including across Europe. This suggests that: a) both techniques are perhaps more accurate than previously thought and b) a large percentage of the non-linear variations seen in our GPS time series are most likely related to geophysical processes rather than analysis error. Low correlation at some sites suggests that local processes or site specific analysis errors dominate the GPS deformation estimates rather than the broad-scale hydrologic signals detected by GRACE. Citation: Tregoning, P., C. Watson, G. Ramillien, H. McQueen, and J. Zhang (2009), Detecting hydrologic deformation using GRACE and GPS, Geophys. Res. Lett., 36, L15401, doi:10.1029/2009GL038718.

\section{Introduction}

[2] The seasonal transfer of water between the continents and the oceans generates changes in surface loading which cause an elastic deformation of the surface of the Earth. Such deformation can be estimated if the load and rheological properties of the Earth are known [Farrell, 1972]. The gravitational response of the Earth is detectable by the Gravity Recovery and Climate Experiment (GRACE) since 2002 [e.g., Tapley et al., 2004; Wahr et al., 1998], while the surface deformation can be observed from an analysis of Global Positioning System (GPS) observations [e.g., Davis et al., 2004; Tregoning et al., 2009]. While detectable, estimates of such variations are very difficult - if not impossible - to verify, in part because of the large spatial scale of the GRACE estimates (around $400 \mathrm{~km}$ ) but also because in-situ measurements of all aspects of the hydrological cycle are discrete and extrapolation to large-scale is not always informative.

[3] That it is possible to derive estimates of surface deformation from changes in gravity offers the possibility

\footnotetext{
${ }^{1}$ Research School of Earth Sciences, Australian National University, Canberra, ACT, Australia.

${ }^{2}$ Surveying and Spatial Science Group, School of Geography and Environmental Studies, University of Tasmania, Hobart, Tasmania, Australia.

${ }^{3}$ Dynamique Terrestre et Planétaire, UMR5562, CNRS, Toulouse, France.
}

Copyright 2009 by the American Geophysical Union. 0094-8276/09/2009GL038718\$05.00 of cross-validating the estimates of water variations from GRACE with ground deformation estimates if the actual variation in the surface can be estimated with sufficient accuracy. Davis et al. [2004] and King et al. [2006] compared annual variations in South America estimated from GPS height time series and from radial displacement derived from GRACE mass anomalies and found high correlation. Bevis et al. [2005] demonstrated high correlation between largeamplitude vertical deformation at a site in the centre of the Amazon Basin and elastic deformation computed from hydrologic signals. On the other hand, poor agreement has been reported in the annual variations in GPS height and GRACE-derived deformation estimates over Europe [van Dam et al., 2007] and Central America [King et al., 2006]. Interpretation of the latter study from a hydrologic perspective is also limited as both the GRACE and GPS data used in that study included deformation induced by atmospheric pressure which has a well known large seasonal component [see, e.g., Tregoning and Watson, 2009].

[4] Over the past decade, considerable effort has been made to reduce the analysis-derived errors in the processing of GPS observations in order to achieve more accurate estimates of site coordinates. Recent improvements in the modelling of tropospheric delays through time-varying mapping functions [e.g., Boehm et al., 2006a], a priori modelling of hydrostatic delays [e.g., Boehm et al., 2006b; Tregoning and Herring, 2006], and better understanding of tidal deformation modelling [Watson et al., 2006] and associated error propagation [e.g., Penna et al., 2007] have led to improvements in the repeatability of coordinate estimates [Tregoning and Watson, 2009]. Of particular interest, many of these advances in analysis strategy have highlighted spurious signals at quasi annual and semi-annual periods (see Tregoning and Watson [2009] for examples relating to mapping functions, a priori hydrostatic delays and atmospheric tidal loading).

[5] However, considerable non-stationary variations remain in GPS time series, particularly (but not only) in the vertical component. Generally, this has been attributed to, for example errors in tropospheric modelling, implying that considerable improvement was still required in the analysis of GPS data. The work by van Dam et al. [2007] used the IGS sinex files and suggested that the difference in annual signals over Europe was probably caused by errors in the analysis of the GPS data. Ray et al. [2008] showed that there are spurious annual and semi-annual periodic signals in GPS time series derived from the same IGS sinex files. It is possible that these may have corrupted the hydrological seasonal signals over Europe and Central America, resulting in the poor agreement found by van Dam et al. [2007] and King et al. [2006].

[6] The purpose of this study is to demonstrate that, in many cases, a large proportion of the non-linear variations in estimated GPS coordinates results from elastic deformation 

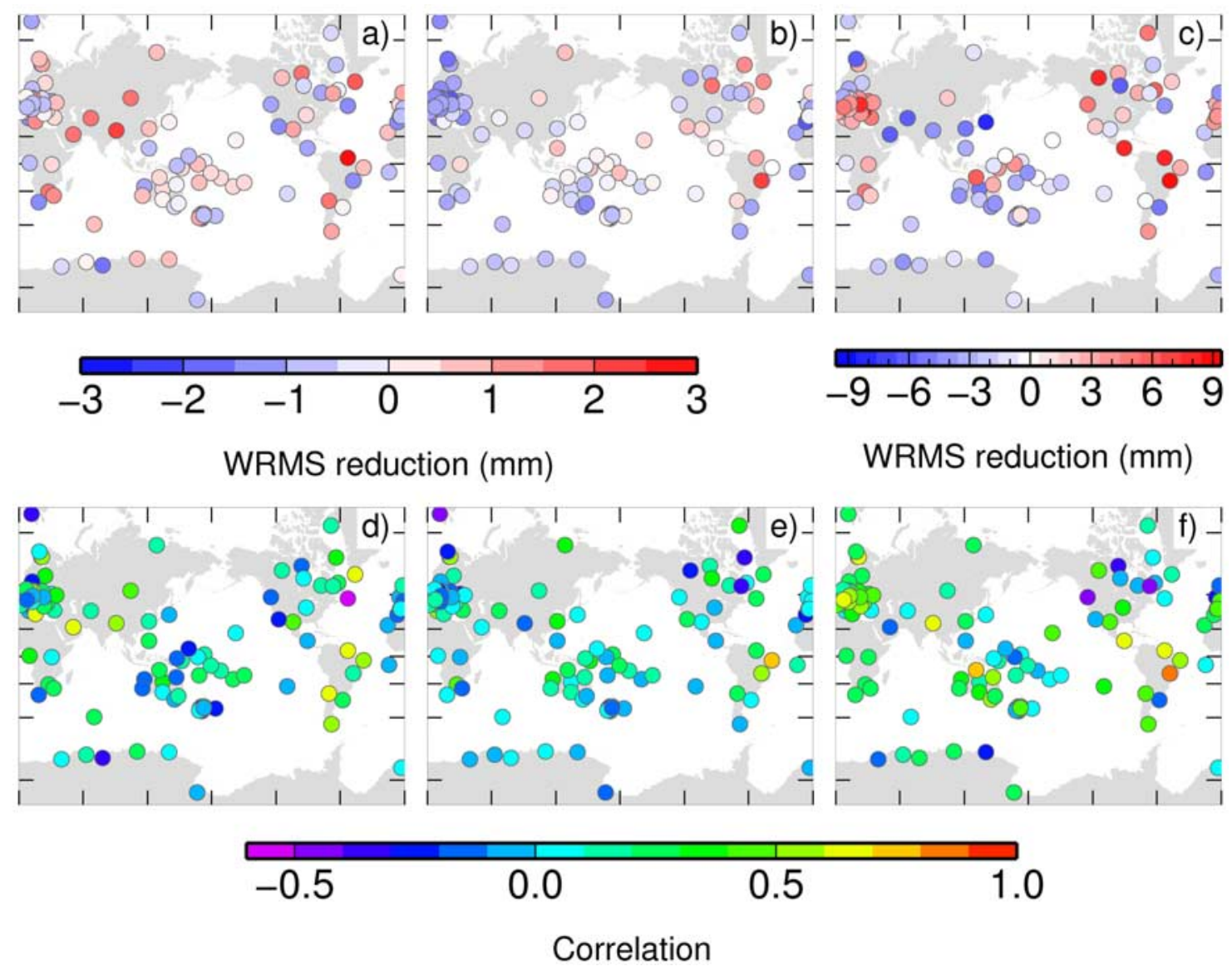

Figure 1. Reduction in RMS of GPS coordinate anomalies after removing a linear trend and elastic deformation derived from GRACE for (a) north, (b) east, (c) up components. Correlations between GPS and GRACE anomalies are shown for (d) north, (e) east, (f) up.

of the Earth's surface caused by hydrologic changes. We show below that this occurs not only in major river basins but also in semi-arid and polar regions. Hydrologic signals are periodic only to a first-order and in this study we look in greater detail at the time series to detect the non-stationary, non-periodic signals across all coordinate components.

\section{GPS Analysis}

[7] We used the GAMIT/GLOBK software [Herring et al., 2008] to generate daily estimates of a global network comprising around 80 sites (Figure 1). Satellite orbits, hourly tropospheric delays, atmospheric gradients (3/day for N/S and $\mathrm{E} / \mathrm{W}$ ) and site coordinates were estimated along with ambiguities and Earth orientation parameters. We used the VMF1 mapping function [Boehm et al., 2006a] and a priori hydrostatic delays derived from the ECMWF numerical weather model [Boehm et al., 2006a] along with non-tidal atmospheric pressure loading [Tregoning and Watson, 2009] and diurnal and semi-diurnal tidal atmospheric pressure loading deformation using the model of Ponte and Ray [2002]. We transformed our daily free-network solutions onto ITRF2005 with a 6-parameter transformation (3 translations, 3 rotations) using the coordinates of 35 sites. Data from 2000-2008 were analysed and are the same solutions interpreted by Tregoning and Watson [2009].
[8] The GPS coordinate estimates include long-term linear velocities resulting from geophysical phenomena not related to elastic deformation (e.g., continental drift in the horizontal coordinates and glacial isostatic adjustment in the vertical component). Therefore, we first remove a linear trend from each component of the GPS time series to generate coordinate 'anomalies'. These are then averaged to the 10-day epochs of the GRACE solutions before comparing with elastic deformation estimates derived from the anomalies of the GRACE estimates of surface loads.

\section{GRACE Analysis}

\subsection{Surface Mass Variation Estimates}

[9] We used RL01 of the temporal gravity field estimates of the Groupe de Recherche en Géodesie Spatiale (GRGS) [Lemoine et al., 2007] which are expressed as spherical harmonic fields to degree and order 50 (spatial resolution of $\sim 400 \mathrm{~km}$ ). As a result of the constraints applied during the process of estimating the coefficients of the spherical harmonic model, these solutions do not require any subsequent filtering or scaling. The solutions have atmospheric mass variations removed (derived from the ECMWF 6-hourly fields) as well as barotropic ocean mass variations (derived from the MOG2D-G model [Carrère and Lyard, 2003]). Readers are referred to Lemoine et al. [2007] for full 

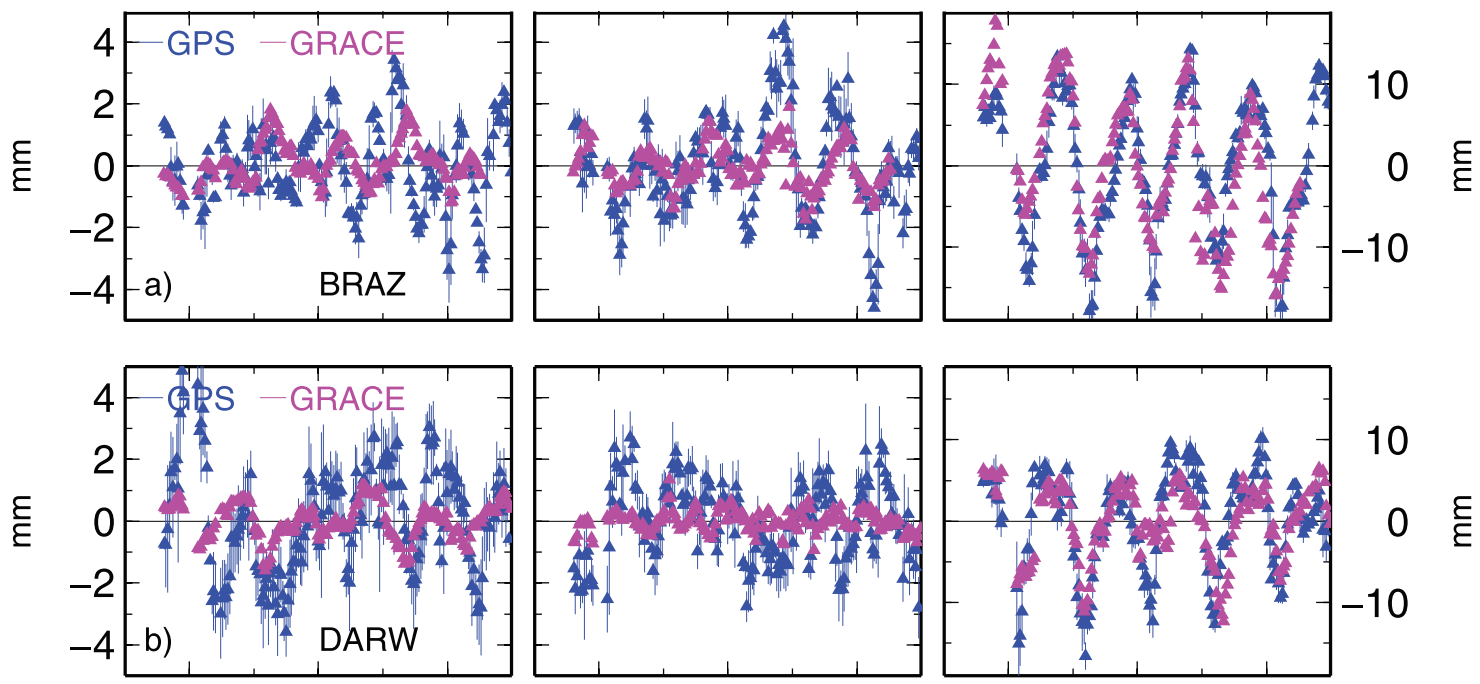

10
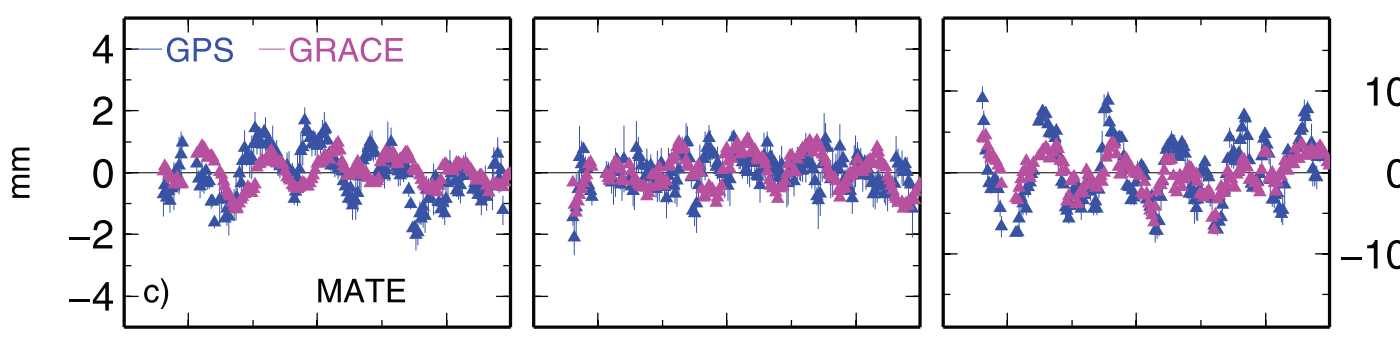

10

$0 \quad \varepsilon$
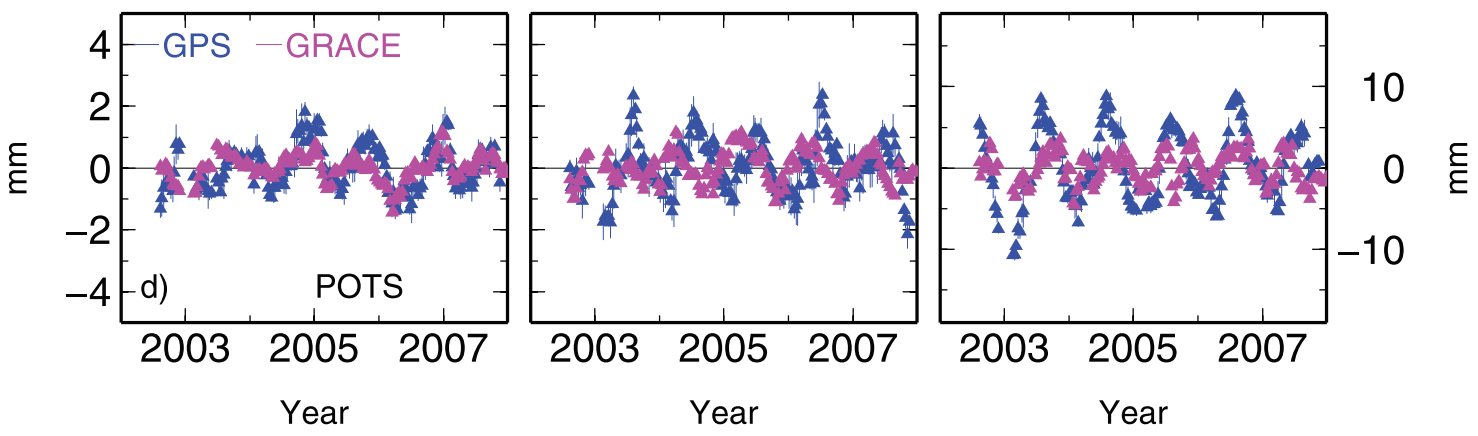

Figure 2. Time series of GPS coordinate anomalies (blue) with elastic deformation computations superimposed as derived from GRACE surface load estimates (pink) for (a) Brasilia, Brazil (b) Darwin, Australia (c) Matera, Italy and (d) Potsdam, Germany.

details of the analysis methods. We removed the mean value of each coefficient of the spherical harmonic models to generate anomaly coefficients. We then converted the spherical harmonic models into surface loads in terms of equivalent water heights [e.g., Wahr et al., 1998]. We inserted degree-1 terms into the GRGS spherical harmonic fields [Munekane, 2007] to make them compatible with the GPS reference frame.

[10] Elastic deformation caused by tidal and non-tidal atmospheric pressure variations have already been accounted for in the GPS analysis [Tregoning and Watson, 2009] but any ocean mass changes that are not tidal in origin (i.e., not modelled by the FES2004 ocean tide loading model [Lyard et al., 2006]) will affect the GPS site coordinate estimates, in particular for coastal sites. Therefore, we reinserted the MOG2D-G model into the GRGS GRACE solutions before computing the mass anomalies. Thus, while the GRACE mass anomalies are corrected for atmospheric pressure effects, they include a component of mass variation that is not hydrologic in nature and are therefore compatible with the GPS height estimates. This phenomenon can be significant, for example the $\sim 0.5 \mathrm{~m}$ annual 'tide' seen in the Gulf of Carpentaria in northern Australia [e.g., Tregoning et al., 2008].

\subsection{Elastic Deformations}

[11] We convolved the mass anomalies derived from the GRACE solutions with the elastic Green's functions of Pagiatakis [1990] to generate estimates of horizontal and vertical deformation [e.g., Farrell, 1972]. We convolved the anomalies on a $1^{\circ} \times 1^{\circ}$ grid covering the entire Earth (we obtain the same results if we use a $0.25^{\circ}$ grid) and the resulting modelled deformations can be compared with the actual movements of GPS sites. If the geophysical processes causing non-linear motions at GPS sites are dominated by 


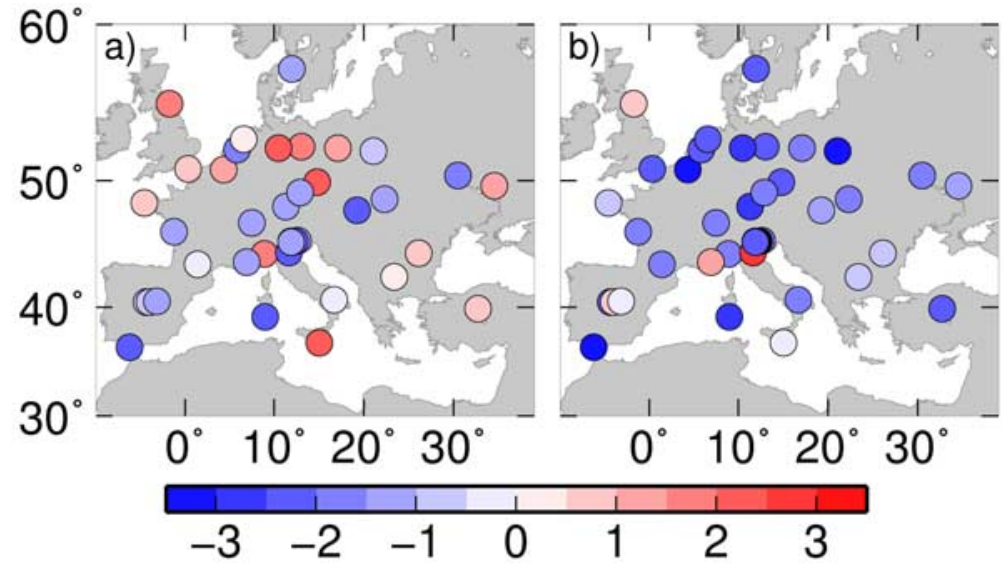

WRMS reduction $(\mathrm{mm})$

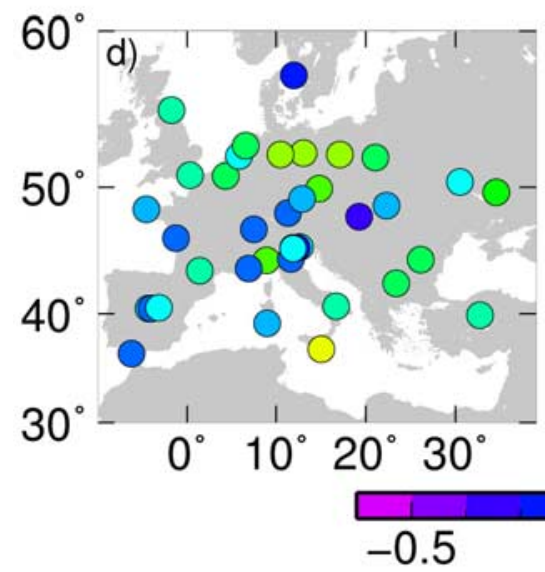

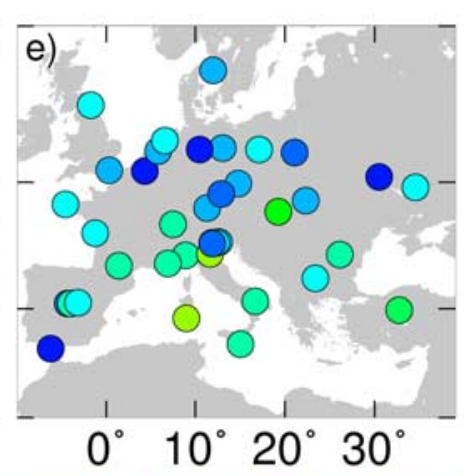

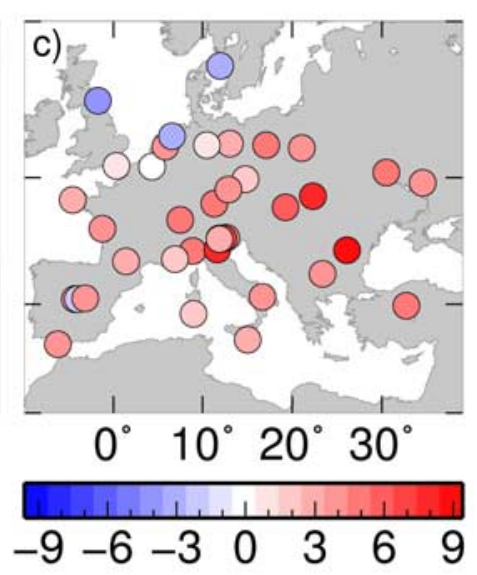

WRMS reduction $(\mathrm{mm})$

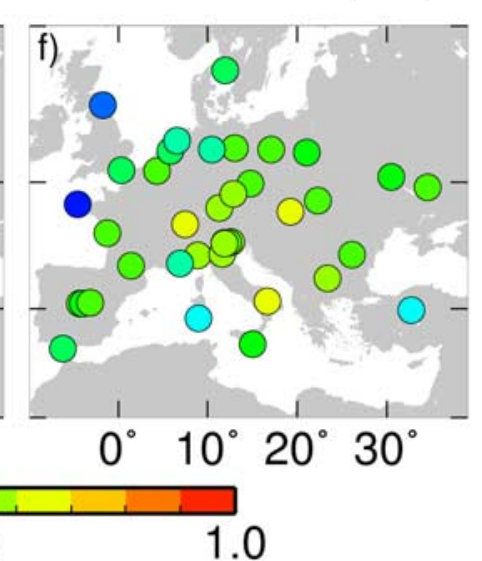

Correlation

Figure 3. Reduction in RMS over Europe of GPS height anomalies after removing a linear trend and elastic deformation derived from GRACE for the (a) north (b) east (c) up components. Correlations are shown for (d) north (e) east and (f) up.

large-scale hydrology then we should expect to see significant correlation between the estimates from GRACE and GPS.

\section{Results}

[12] We subtracted the GRACE-derived elastic deformation computations from the GPS coordinate anomalies and computed the (in quadrature) reduction in weighted root mean square (WRMS) (Figure 1). It is immediately apparent that there is a mix of significant improvements as well as cases where the WRMS is increased by this process. Unsurprisingly, the most significant reductions occur in regions of known large-amplitude hydrologic processes (e.g., the Amazon Basin in South America).

[13] The correlation between GRACE-derived deformation and GPS estimated deformation is shown in Figures 1d-1f. Many sites show positive correlations, which is more convincing in the vertical component. In fact, while cases of very high correlation are found in the north and east components (e.g., in eastern South America) there are some sites with negative correlation and some sufficiently negative to indicate a strong anti-correlation or phase difference between observed signals.
[14] Figure 2 shows the time series of north, east and vertical components of four sites, along with the computed elastic deformation from the GRACE water anomaly estimates. The correlations at BRAZ (located on the edge of the Amazon Basin in Brasila) between the GPS coordinate anomalies and the GRACE-derived elastic deformation anomalies are $0.1,0.6$ and 0.9 for $\mathrm{N}, \mathrm{E}, \mathrm{U}$ respectively. Despite the formal uncertainties of $2 \mathrm{~mm}$ on the horizontal coordinate estimates, variations in east often track the elastic deformation computations to within $\sim 1 \mathrm{~mm}$. This inspires confidence in the improved accuracy of both the GPS and the GRACE analyses. The correlations seen at Darwin are $0.2,0.2$ and 0.7 for $\mathrm{N}, \mathrm{E}$ and $\mathrm{U}$, respectively.

[15] The work by van Dam et al. [2007] found that removing GRACE-derived elastic deformation estimates from GPS height anomaly time series in Europe reduced the RMS at only three sites and suggested that the poor agreement between GPS and GRACE annual variations was likely caused by inaccuracies in the GPS analysis, for example in the propagation of ocean tide loading errors. We find a reduction in RMS over Europe at 32 of 36 sites analysed (Figure 3). A visual comparison of their GRACE and GPS time series with ours shows that the significant difference lies in the GPS rather than the GRACE results. The 
correlation in height at Matera and Potsdam is 0.6 and 0.4 , respectively (Figure 2) and the agreement in phase of the anomaly signals is good in north but poor in east. This is typical of sites in Europe (Figure 3). There may even be a north/south trend in the correlation in the east component, although the evidence for this is weak.

[16] It is clear from Figures 1 and 3 that, in many cases, a considerable reduction in the variance of the residuals is achieved by accounting for the elastic deformation associated with fluid mass changes. However, this is not always the case - indeed the variance is increased at $48 \%, 71 \%$ and $47 \%$ of the sites (for N, E, U, respectively) when correcting with the GRACE-derived estimates.

\section{Discussion and Conclusions}

[17] Our comparison of GPS coordinate anomalies and GRACE-derived elastic deformation estimates shows far greater agreement, and therefore improvement in the RMS of the difference, when compared with the findings of van Dam et al. [2007] and King et al. [2006]. Thus, their conclusion that the lack of correlation was caused by errors in the GPS analysis was probably correct. We suggest that the use in our GPS analysis of the latest strategies that are known to reduce spurious signals at quasi-annual and semiannual periods (notably those produced through using inadequate mapping functions, a priori zenith hydrostatic delays and failing to model atmospheric pressure tidal loading [Tregoning and Watson, 2009]) has led to improved accuracy of our GPS solutions. We also find reductions in RMS of the horizontal coordinates, especially in regions close to large continental hydrology processes such as the Amazon Basin. This leads to increased confidence in both the GPS and GRACE analyses used in this study.

[18] However, we find many examples where removing the GRACE-derived elastic deformation computations actually increases the RMS of the coordinate time series, in particular in the horizontal component. In many cases this is due to a phase difference between the two solutions which may still be symptomatic of residual spurious signals in the GPS solutions. We do not have strong explanations for the examples of poor agreement but suggest that it might also be caused by a geophysical process that is affecting the two observation techniques differently. For example, a local hydrological process might affect a GPS time series significantly but be too small spatially to be detected by GRACE.

[19] We find a higher correlation between GPS and GRACE anomalies in the vertical component than in the horizontal components, even though seasonal variations are often evident in the latter in both techniques but not necessarily in phase. This might be due to the increased sensitivity of the horizontal deformation to the spatial location of the water loads where destructive interference can occur between loads in opposite directions, whereas all loads will add constructively in the computation of the vertical deformation. This might indicate errors in the spatial distribution of water loads as estimated by GRACE as a result of the nominal spatial resolution of $\sim 400 \mathrm{~km}$ of the GRACE solutions. Another possibility is residual spurious signals in the GPS solution, arising from, for example residual mismodelled high frequency signals, near field multipath or quasi periodic errors in the frame realisation process.

[20] Acknowledgments. We thank the GRGS GRACE team for making their 10-day solutions freely available, the IGS for providing global GPS data and two reviewers for helpful comments. The GPS data were analysed on the Terrawulf II computational facility at the Research School of Earth Sciences, a facility supported through the AuScope initiative. AuScope Ltd is funded under the National Collaborative Research Infrastructure Strategy (NCRIS), an Australian Commonwealth Government Programme. This research was supported under the Australian Research Council's Discovery Projects funding scheme (DP0877381, DP0985080).

\section{References}

Bevis, M., D. Alsdorf, E. Kendrick, L. P. Fortes, B. Forsberg, R. Smalley Jr., and J. Becker (2005), Seasonal fluctuations in the mass of the Amazon River system and Earth's elastic response, Geophys. Res. Lett., 32, L16308, doi:10.1029/2005GL023491.

Boehm, J., B. Werl, and H. Schuh (2006a), Troposphere mapping functions for GPS and very long baseline interferometry from European Centre for Medium-Range Weather Forecasts operational analysis data, J. Geophys. Res., 111, B02406, doi:10.1029/2005JB003629.

Boehm, J., A. Niell, P. Tregoning, and H. Schuh (2006b), Global Mapping Function (GMF): A new empirical mapping function based on numerical weather model data, Geophys. Res. Lett., 33, L07304, doi:10.1029/ 2005GL025546.

Carrère, L., and F. Lyard (2003), Modeling the barotropic response of the global ocean to atmospheric wind and pressure forcing: Comparisons with observations, Geophys. Res. Lett., 30(6), 1275, doi:10.1029/ 2002GL016473

Davis, J. L., P. Elósegui, J. X. Mitrovica, and M. E. Tamisiea (2004), Climate-driven deformation of the solid Earth from GRACE and GPS, Geophys. Res. Lett., 31, L24605, doi:10.1029/2004GL021435.

Farrell, W. E. (1972), Deformation of the Earth by surface loads, Rev. Geophys., 10, 761-797.

Herring, T. A., R. W. King, and S. C. McClusky (2008), Introduction to GAMIT/GLOBK, Mass Inst. of Technol., Cambridge.

King, M., P. Moore, P. Clarke, and D. Lavallée (2006), Choice of optimal averaging radii for temporal GRACE gravity solutions, a comparison with GPS and satellite alitmetry, Geophys. J. Int., 166, 1-11.

Lemoine, J.-M., S. Buinsma, S. Loyer, R. Biancale, J.-C. Marty, F. Perosanz, and G. Balmino (2007), Temporal gravity field models inferred from GRACE data, Adv. Space Res., 39, 1620-1629.

Lyard, F., F. Lefèvre, T. Letellier, and O. Francis (2006), Modelling the global ocean tides: A modern insight from FES2004, Ocean Dyn., 56, $394-415$.

Munekane, H. (2007), Ocean mass variations from GRACE and tsunami gauges, J. Geophys. Res., 112, B07403, doi:10.1029/2006JB004618.

Pagiatakis, S. D. (1990), The response of a realistic Earth to ocean tide loading, Geophys. J. Int., 103, 541-560.

Penna, N. T., M. A. King, and M. P. Stewart (2007), GPS height time series: Short-period origins of spurious long-period signals, J. Geophys. Res., 112, B02402, doi:10.1029/2005JB004047.

Ponte, R. M., and R. D. Ray (2002), Atmospheric pressure corrections in geodesy and oceanography: A strategy for handling air tides, Geophys. Res. Lett., 29(24), 2153, doi:10.1029/2002GL016340.

Ray, J., Z. Altamimi, X. Collieux, and T. van Dam (2008), Anomalous harmonics in the spectra of GPS position estimates, GPS Solutions, 12, 55-64, doi:10.1007/s10291-007-0067-7.

Tapley, B. D., S. Bettadpur, M. Watkins, and C. Reigber (2004), The gravity recovery and climate experiment: Mission overview and early results, Geophys. Res. Lett., 31, L09607, doi:10.1029/2004GL019920.

Tregoning, P., and T. A. Herring (2006), Impact of a priori zenith hydrostatic delay errors on GPS estimates of station heights and zenith total delays, Geophys. Res. Lett., 33, L23303, doi:10.1029/2006GL027706.

Tregoning, P., and C. Watson (2009), Atmospheric effects and spurious signals in GPS analyses, J. Geophys. Res., doi:10.1029/2009JB006344, in press.

Tregoning, P., G. Ramillien, and K. Lambeck (2008), GRACE estimates of sea surface height anomalies in the Gulf of Carpentaria, Australia, Earth Planet. Sci. Lett., 271, 241-244, doi:10.1016/j.epsl.2008.04.018.

Tregoning, P., G. Ramillien, H. McQueen, and D. Zwartz (2009), Glacial isostatic adjustment and non-stationary signals observed by GRACE, J. Geophys. Res., 114, B06406, doi:10.1029/2008JB006161.

van Dam, T., J. Wahr, and D. Lavallée (2007), A comparison of annual vertical crustal displacements from GPS and Gravity Recovery and Climate Experiment (GRACE) over Europe, J. Geophys. Res., 112, B03404, doi:10.1029/2006JB004335 
Wahr, J., M. Molenaar, and F. Bryan (1998), Time variability of the Earth's gravity field: Hydrological and oceanic effects and their possible detection using GRACE, J. Geophys. Res., 103, 30,205-30,229.

Watson, C., P. Tregoning, and R. Coleman (2006), Impact of solid Earth tide models on GPS coordinate and tropospheric time series, Geophys. Res. Lett., 33, L08306, doi:10.1029/2005GL025538.

H. McQueen, P. Tregoning, and J. Zhang, Research School of Earth Sciences, Australian National University, Mills Road, Canberra, ACT 0200, Australia. (paul.tregoning@anu.edu.au)
G. Ramillien, Dynamique Terrestre et Planétaire, UMR 5562, CNRS, 14 Avenue Edouard Berlin, F-31400 Toulouse CEDEX, France.

C. Watson, Surveying and Spatial Science Group, School of Geography and Environmental Studies, University of Tasmania, Private Bag 76, Hobart, Tas 7001, Australia. 